\title{
Electrical bioimpedance as a diagnostic method for lipodystrophy syndrome in HIV-infected patients in Lower Silesia, Poland
}

\author{
Justyna Drelichowska ${ }^{1,2}$, Wiesława Kwiatkowska ${ }^{1,2}$, Brygida Knysz ${ }^{1,3}$, Szymon Manasterski ${ }^{4}$, \\ Marcin Czarnecki ${ }^{1,3}$, Jacek Gąsiorowski ${ }^{1,3}$, Maciej Karczewski ${ }^{1}$, Wojciech Witkiewicz ${ }^{1,2}$ \\ ${ }^{1}$ Wrovasc - Integrated Cardiovascular Centre, Regional Specialist Hospital, Research and Development Center in Wroclaw, Poland \\ ${ }^{2}$ Department of Angiology, Regional Specialist Hospital in Wroclaw, Research and Development Center in Wroclaw, Poland \\ ${ }^{3}$ Department of Infectious Diseases, Wroclaw Medical University, Poland \\ ${ }^{4}$ Department of Traumatology and Hand Surgery, University Hospital in Wroclaw, Poland
}

\begin{abstract}
Introduction: Despite a great progress in antiretroviral therapy (ARV) there are many human immunodeficiency virus (HIV)-infected patients with lipodystrophy syndrome (LS). However, there are still no clearly defined criteria to diagnose dystrophic changes. It would be valuable to introduce a non-invasive and inexpensive method to clinical practice, which could objectify the assessment of LS in HIV-infected population. The aim was to determine usefulness of electrical bioimpedance in the diagnostics of lipodystrophy syndrome.

Material and methods: One hundred and ten HIV-infected patients, on antiretroviral therapy, were examined for the presence of LS. The control group included 42 healthy individuals. The study protocol included anthropometric measurements and bioelectrical impedance analysis (BIA). The measurements of BIA were interpreted using specific computer software. All parameters were subjected to a comparative analysis between the study group and the control group.

Results: HIV-infected patients had significantly higher percentage values of fat-free mass (FFM), total body water (TBW) and muscle mass (MM) than the control group. Fat mass (FM) and percentage content of fat tissue (FM\%) was significantly lower in the study group. A linear relationship of FM, percentage content of fat-free mass (FFM\%), percentage content of total body water (TBW\%) and percentage content of muscle mass (MM\%) upon the severity of LS in HIV-positive patients has been demonstrated. The most significant changes were observed in patients with lipoatrophy who had the lowest absolute and percentage values of FM and the highest values of FFM\%, MM\% and TBW\%.

Conclusions: The results support the potential usefulness of BIA as a non-invasive and low-cost diagnostic method for LS. Parameters such as FM\%, FFM\%, and TBW\% can be used to assess the severity of lipodystrophy. However, these parameters require a larger study population and continuation of research.
\end{abstract}

HIV AIDS Rev 2017; 16, 2: 70-76

DOI: https://doi.org/10.5114/hivar.2017.66795

Key words: HIV, bioelectrical impedance analysis, antiretroviral therapy, lipodystrophy syndrome.

Address for correspondence: Brygida Knysz, Wrovasc Integrated Cardiovascular Centre, Regional Specialist Hospital, Research and Development Center in Wroclaw, 73a H. Kamienskiego St., 51-124 Wroclaw, Poland, phone: +48 6030766 48, e-mail: brygida.knysz@gmail.com
Article history:

Received: 08.07.2016

Received in revised form: 30.07 .2016

Accepted: 06.01.2017

Available online: 24.03 .2017
International Journal of HIV-Related Problems

HIV \& AIDS

R e vi e w 


\section{Introduction}

Despite a great progress in the antiretroviral therapy (ARV) there are still many human immunodeficiency virus (HIV)-positive patients with lipodystrophy syndrome (LS), including metabolic disturbances, significant changes in body composition, resulting in worsening the quality of life of these patients $[1,2]$. An early detection of dystrophic changes in patients undergoing antiretroviral treatment may allow a quick response and modification of therapy in order to inhibit further development of disorders [3].

There are several research methods used in the diagnostics of LS. These include dual-energy X-ray absorptiometry (DXA), bioelectrical impedance analysis (BIA) and, occasionally, computed tomography and magnetic resonance imaging [4-9]. Scanning the whole body using DXA is considered a reference method for the diagnosis of LS. This is an objective method that enables a precise assessment of parameters, such as fat-free mass (FFM), fat mass (FM) and bone mineral content [5-7]. The disadvantage of this procedure is its high cost and low availability for routine use.

Bioelectrical impedance analysis is becoming increasingly widespread due to its low cost, ease of performing and non-invasiveness. It enables reliable analysis of body composition in healthy subjects, and it is increasingly being used in the assessment of pathologies associated with body composition in patients with obesity and cachexia. In several clinical studies, the BIA technique has been shown to be a useful and objective method of examining HIV-infected patients, with FFM (mass of internal organs and muscles) and FM $[5,6,8]$. There are also studies in which it was concluded that the BIA test is not an appropriate method for assessing fat tissue redistribution in patients infected with HIV [10].

The aim of the study was to determine usefulness of electrical bioimpedance in the diagnostics of lipodystrophy syndrome.

\section{Material and methods}

It was an observational study. Two groups of patients were included: HIV-positive and control group. These subjects were part of groups described in our previous publications [11]. One hundred and ten HIV-infected, Caucasian individuals, treated at the Acquired Immunodeficiency Syndrome Out-Patient Clinic in Wroclaw (living in the Lower Silesia region, Poland) (average age $39.8 \pm 9.3$; men $64 \%$, women $36 \%$ ), with known virological and immune status were examined. The inclusion criteria were: documented HIV infection and antiretroviral therapy (cART) at least for 2 years. The exclusion criteria were: AIDS diagnosis, acute medical condition (fever, severe infection, inflammation), serum creatinine level over $2 \mathrm{mg} / \mathrm{dl}$ and an over fivefold increase in alanine aminotransferase level (ALT). All patients have been on CART for 2-8 years (4 years on average). They got cART consisting of nucleotide reverse transcriptase inhibitors (NRTIs) and protease inhibitors (PIs) or non-nucle- oside reverse transcriptase inhibitor (NNRTI), NRTI, NtRTI and PIs or NNRTI. The treatment regimens were changed before entering the study in many patients because of the lack of viral efficacy or drug toxicity. Because of a small number of data, analysis concerning influence of special drugs and their classes on LS was not done. The time of cumulative antiretroviral treatment with medications from all antiretroviral classes was from 8 to 25 years (16.6 years on average); the longest cumulative time of treatment concerned nucleoside and nucleotide reverse transcriptase inhibitors (NRTIs, NtRTI).

The control group included 42 healthy individuals, matched for age and sex with the patients, with negative medical history of chronic diseases and cardiovascular events, recruited contemporaneously (average age $41 \pm 11.5$, proportion of men $62 \%$, women $38 \%$ ). These healthy subjects were recruited at random through two general practices in the Lower Silesia region.

Following the severity of LS, HIV-infected patients were divided into two main subgroups:

1) patients with no symptoms or slight features of lipodystrophy (noticeable only if specifically looked for, with no change in clothing fit) - 57 individuals - LS1,

2) patients with moderate (easily noted by the patient or physician, often requiring a change in clothing size) and severe changes (obvious to the casual observer, requiring a change in clothing size) - 53 individuals - LS2.

The electrical bioimpedance test was performed with the use of bioelectrical impedance analyser Quantum II with alligator clips using dedicated electrodes on a non-conductive examination couch. The measurements were done by the same person - a specialized doctor. The patients were fasted in the supine position, with no contact with any metal, motionless, with abducted upper limbs (extending away from the body) at an angle of $30^{\circ}$ and legs apart. The study involved the right side of the patient's body. After preparing the skin of the hand and foot (cleaned and degreased), two electrodes (Welch Allyn) were attached to the right hand and two electrodes to the right foot, and the electrodes were fastened with alligator clips. Next, the analyser was turned on and the resistance measurement mode was launched, followed by the reactance mode. Readings were recorded on the patient's form. The numeric data were interpreted using computer software BodyGram by Akern S.r.l.

All parameters were subjected to a comparative analysis between the study group and the control group, and between subgroups. Parameters significantly discriminating groups and subgroups were subjected to further analysis, such as correlations with anthropometric variables, markers of subclinical atherosclerosis, and antiretroviral treatment.

The following parameters were assessed:

- FFM (mass of internal organs and muscles),

- FM,

- TBW (mainly reflects FFM),

- extracellular water (ECW),

- intracellular water (ICW; mainly in muscles and internal organs, and to a lesser extent in fat tissue), 
- body cell mass (BCM; mainly mass of internal organs and muscles excluding fat tissue),

- muscle mass (MM),

- phase angle - a measure of the electrical phase shift caused by the "delay" resulting from the resistance associated with electrical capacitance of cell membranes, which ranges between $0^{\circ}$ and $90^{\circ}$ [12].

The following parameters were also included in analysis: age, sex, pack-years (smoking cigarettes), diagnosed hypertension, insulin resistance using homeostasis model assessment (HOMA-IR), and cumulative treatment time of ART.

\section{Statistical analysis}

The normality test was performed using D'Agostino-Pearson method. Due to the lack of fit, the analyses were performed using nonparametric tests. The quantitative variables were presented as the median with the interquartile range and qualitative variables - as sizes and percentages. The differences between two quantitative variables were analyzed using the Mann-Whitney test. Spearman's rank correlation coefficient was used to measure relationships between quantitative variables. Logistic regression was also conducted on the impact of selected BIA parameters and selected risk factors for the occurrence of advanced lipodystrophy syndrome. Additionally, a stepwise regression procedure was used. Results with a significance level of $p<0.05$ were found significant. The analysis was performed using R-3.1.1 for Windows and MedCalc for Windows (version 14).

All participants of the study provided their written informed consent to participate in this study, according to the Helsinki Declaration, and the study was approved by the Commission of Bioethics at the Regional Specialist Hospital in Wroclaw, Research and Development Centre in Wroclaw (permission number: KB/nr/10/2014).

\section{Results}

Noticeable differences between the two groups included lower BMI and significant deviations in blood lipid concentration in the study group, where high concentration of tri- glycerides and low concentration of all cholesterol fractions, were observed (Table 1). In the study group, a significantly lower hip diameter was observed $(p<0.0001)$, which had an impact on the higher waist to hip ratio (WHR) value. However, the differences between the patients' and controls' WHR were of little statistical significance. HIV-positive patients much more frequently had proper body weight or were underweight and were significantly less frequently overweight (Table 1).

In the analysis of body composition using bioimpedance, a comparison of the study group with the control group showed significantly higher percentage values of FFM, TBW and MM in HIV-patients $(p<0.0001, p<0.0001, p=0.014$, respectively; Table 2 ). The value of FM - both absolute and as a percentage - was significantly lower in the study group $(p<0.0001)$. The remaining parameters of electrical bioimpedance (FFM, TBW, ECW, BCM, MM, phase angle, and percentage values of ECW, ICW, BCM) were not significantly different between the two groups.

Comparing the bioimpedance parameters between two subgroups, LS1 and LS2, significantly higher values of percentage content of fat tissue (FM\%), total body water (TBW\%) and muscle mass (MM\%), and at the same time significantly lower absolute and percentage values of FM were shown in subgroup LS2 (Table 3). In a further analysis comparing subgroups LS1 and LS2 with the control group, significantly higher values of MM\% were shown in infected patients in subgroup LS2 compared to uninfected individuals $(p=0.005)$. In terms of the other investigated parameters of the body composition, no significant differences between subgroups were established.

When analysing BIA parameters depending on the type of lipodystrophy disorder, differences were observed. The most significant changes were observed in the values of fat mass. The lowest FM values were recorded in a subgroup of patients with lipoatrophy compared with patients with lipoaccumulation $(p<0.0001)$, with mixed changes $(p=0.011)$ and those without LS $(p=0.048)$. Furthermore, the FM value was significantly higher in patients with lipoaccumulation compared with patients with a mixed type of LS $(p=0.037)$. Similar trends were also observed in the percentage value of FM. The percentage values of FFM were

Table 1. Characteristics of the study group (SG) and the control group (CG)

\begin{tabular}{l|c|c|c}
\hline Feature & $\mathrm{SG}, n=110$ & $\mathrm{CG}, n=42$ & $p$-value \\
\hline $\mathrm{BMI}^{*}$ & $22.86(21-25)$ & $25.6(24-27)$ & 0.0001 \\
\hline $\mathrm{WHR}^{*}$ & $0.90(0.84-0.96)$ & $0.89(0.78-0.94)$ & 0.055 \\
\hline $\mathrm{TC}(\mathrm{mg} / \mathrm{dl})(\mathrm{a}, \mathrm{sd})$ & $195.30 \pm 45.40$ & $212.67 \pm 38.70$ & 0.03 \\
\hline $\mathrm{LDL}(\mathrm{mg} / \mathrm{dl})(\mathrm{a}, \mathrm{sd})$ & $110.13 \pm 38.10$ & $126.34 \pm 31.70$ & 0.016 \\
\hline $\mathrm{HDL}(\mathrm{mg} / \mathrm{dl}){ }^{*}$ & $51(40-64)$ & $58.5(47-71)$ & 0.012 \\
\hline $\mathrm{TG}(\mathrm{mg} / \mathrm{dl}){ }^{*}$ & $131.5(93-189)$ & $100.5(68-137)$ & 0.0077 \\
\hline Obesity/overweight/underweight, $n(\%)$ & $6 / 21 / 7(5.5 / 19.1 / 6.3)$ & $5 / 23 / 0(12 / 54.8 / 0)$ & 0.0001 \\
\hline
\end{tabular}

${ }^{*}$ Median, $I Q R, n(\%)$ - absolute number (percentage), other data - arithmetic mean

$B M I$ - body mass index, WHR - waist to hip ratio, TC - total cholesterol, LDL - low-density lipoprotein, HDL - high-density lipoprotein, TG - triglyceride 
Table 2. Comparison of electrical bioimpedance parameters between the study and control groups (Mann-Whitney test)

\begin{tabular}{l|c|c|c}
\hline Variable & SG, $n=110$ & CG, $n=42$ & $p$-value \\
\hline FM & $11.35(7.2-16.4)$ & $16.6(14.6-22.5)$ & 0.0001 \\
\hline FFM & $57.75(49-64.9)$ & $61.1(48.4-66.7)$ & 0.16 \\
\hline TBW & $42.25(36.2-47.5)$ & $45.3(37.7-48.8)$ & 0.13 \\
\hline ECW & $16.95(14.9-19.5)$ & $17.8(16.1-19.2)$ & 0.4 \\
\hline BCM & $32.75(27.5-38.8)$ & $36.45(29.4-42.1)$ & 0.13 \\
\hline MM & $39.85(33.8-47.1)$ & $44.3(35.9-50.6)$ & $<0.0001$ \\
\hline FM (\%) & $17.15(12.6-22.4)$ & $22.75(19.2-27.8)$ & $<0.0001$ \\
\hline FFM (\%) & $82.85(77.6-87.4)$ & $77.25(72.2-80.8)$ & $<0.0001$ \\
\hline TBW (\%) & $60.65(57.2-64.5)$ & $56.75(53.6-59.4)$ & 0.096 \\
\hline ECW (\%) & $41.75(39.3-43.9)$ & $40.7(39.3-42.8)$ & 0.099 \\
\hline BCM (\%) & $58.15(55.7-60.9)$ & $59.25(56.9-60.9)$ & 0.014 \\
\hline MM (\%) & $58.1(52.7-63.1)$ & $55.45(51.4-59.2)$ & 0.096 \\
\hline ICW (\%) & $58.25(56.1-60.7)$ & $59.3(57.2-60.7)$ & 0.11 \\
\hline Phase angle & $6.95(6.4-7.6)$ & $7.2(6.7-7.6)$ & \\
\hline
\end{tabular}

FM - fat mass, FFM - fat-free mass, TBW - total body water, ECW - extracellular water, BCM - body cell mass, MM - muscle mass, FM (\%) - percentage content of fat tissue, FFM (\%) - percentage content of fat-free mass, TBW (\%) - percentage content of total body water, ECW (\%) - percentage content of extracellular water, BCM (\%) - percentage content of body cell mass, MM (\%) - percentage content of muscle mass, ICW (\%) - percentage content of intracellular water

Table 3. Parameters of bioimpedance - a comparison of statistically significant differences between the studied subgroups LS1 and LS2 (Mann-Whitney test)

\begin{tabular}{l|c|c|c}
\hline Variable & LS 1 & LS2 & $p$-value \\
\hline FM & $12.8(8.8-18.1)$ & $9.7(7.1-13.6)$ & 0.022 \\
\hline FM (\%) & $18.9(13.2-25.6)$ & $15.2(11.9-20.2)$ & 0.029 \\
\hline FFM (\%) & $81.1(74.4-86.8)$ & $84.8(79.8-88.0)$ & 0.029 \\
\hline TBW (\%) & $59.3(54.7-63.6)$ & $62(58.8-65.4)$ & 0.018 \\
\hline MM (\%) & $57.4(51.5-62.2)$ & $59.7(54.9-64.1)$ & 0.049
\end{tabular}

FM - fat mass, FM (\%) - percentage content of fat tissue, FFM (\%) - percentage content of fat-free mass, TBW-total body water, MM - muscle mass

highest in patients with lipoatrophy compared with patients from the lipoaccumulation subgroup $(p<0.0001)$ and those with mixed changes $(p=0.025)$. Percentage muscle mass reached its peak value in the subgroup with atrophic changes compared to patients with a mixed type of LS $(p=0.014)$. The percentage of total body water reached its peak value in patients with atrophic changes in comparison with patients with accumulation, with mixed changes and those patients without LS ( $p<0.0001, p=0.01, p=0.046$, respectively).

Table 4 presents results of a correlation analysis of selected, statistically significant BIA parameters with chosen anthropometric characteristics, NRTI treatment and lipodystrophy. There was a positive statistically significant correlation of the value of FM\% with BMI and with waist and hip circumference, but there was no significant relationship with WHR. A negative correlation of TBW\% with BMI was shown and also with waist and hip circumference, but no significant relationship with WHR. The MM\% parameter
Table 4. Significant relationships of electrical bioimpedance parameters with selected anthropometric characteristics and lipodystrophy. Spearman's correlation

\begin{tabular}{l|c|c|c|c}
\hline Variable & rho & 0.528 & -0.54 & -0.229 \\
\hline \multirow{4}{*}{ BMI } & $p$ & $<0.0001$ & $<0.0001$ & 0.0162 \\
\cline { 2 - 5 } & $n$ & 110 & 110 & 110 \\
\hline \multirow{3}{*}{$\begin{array}{l}\text { Waist } \\
\text { circumference }\end{array}$} & rho & 0.226 & -0.254 & -0.007 \\
\cline { 2 - 5 } & $p$ & 0.0178 & 0.0075 & 0.9408 \\
\cline { 2 - 5 } & $n$ & 110 & 110 & 110 \\
\hline \multirow{3}{*}{$\begin{array}{l}\text { Hip } \\
\text { circumference }\end{array}$} & $p$ & $<0.0001$ & $<0.0001$ & 0.0028 \\
\cline { 2 - 5 } & $n$ & 110 & 110 & 110 \\
\hline \multirow{4}{*}{\begin{tabular}{l} 
WHR \\
\cline { 2 - 5 }
\end{tabular}} & rho & -0.053 & 0.028 & 0.16 \\
\cline { 2 - 5 } & $p$ & 0.5803 & 0.7742 & 0.0953 \\
\hline \multirow{3}{*}{$\begin{array}{l}\text { Lipodystrophy } \\
\text { (quantitatively) }\end{array}$} & $n$ & 110 & 110 & 110 \\
\cline { 2 - 5 } & $n$ & -0.257 & 0.267 & 0.202 \\
\cline { 2 - 5 } & $n$ & 110 & 110 & 110 \\
\hline
\end{tabular}

rho-Spearman correlation coefficient, $p$-value, $n$ - number of patients FM (\%) - percentage content of fat tissue, TBW (\%) - percentage content of total body water, MM (\%) - percentage content of muscle mass, BMI-body mass index, WHR - waist to hip ratio

had a negative correlation with BMI and hip circumference. Lipodystrophy, as a quantitatively expressed continuous variable, exhibited a negative correlation with the FM\%, as well as a positive correlation with TBW\% and MM\%. 
Table 5. Risk factors and percentage content of fat tissue (FM\%) as variables in the study of individual impacts on the occurrence of advanced lipodystrophy. Logistic regression

\begin{tabular}{l|c|c|c}
\hline Variable & OR & $95 \% \mathrm{Cl}$ & $p$-value \\
\hline Unadjusted model \\
\hline Age & 1.0598 & 0.9999 to 1.1232 & 0.0504 \\
\hline Sex - Male & 0.715 & 0.2069 to 2.4708 & 0.5959 \\
\hline Pack-years & 1.0205 & 0.9876 to 1.0545 & 0.2258 \\
\hline Hypertension & 1.4134 & 0.4753 to 4.2036 & 0.5338 \\
\hline HOMA-IR* & 1.3987 & 0.8656 to 2.2600 & 0.1705 \\
\hline Cumul. ART & 1.0599 & 1.0140 to 1.1079 & 0.01 \\
\hline FM\% & 0.9209 & 0.8498 to 0.9979 & 0.0443 \\
\hline Stepwise & 1.0336 & 1.0031 to 1.0649 & 0.0304 \\
\hline Pack-years & 1.0672 & 1.0223 to 1.1141 & 0.003 \\
\hline Cumul. ART & 0.9376 & 0.8817 to 0.9970 & 0.0398 \\
\hline FM\% & *HOMA-IR-homeostasis model assessment of insulin resistance \\
Pack-years - smoking cigarettes, ART-antiretroviral therapy, FM (\%) - per- \\
centage content of fat tissue
\end{tabular}

There was no significant relationship between the parameters of electrical bioimpedance and the immune or virological status of patients. Patients with a recognised metabolic syndrome showed significantly lower values of TBW\% and a significantly higher percentage of fat tissue.

In subsequent analyses, we decided to investigate the effect of the BIA parameters that showed the greatest significance in the univariate analysis - i.e. FM\% and TBW\% - on the occurrence of advanced lipodystrophy syndrome. The FFM\% parameter is a reflection of the FM\% parameter, and hence was not included in the analysis, while the MM\% was not included because of the limit of significance in the univariate analysis. The FM\% value, expressed as a percentage of body fat content, had a significant impact on the occurrence of advanced lipodystrophy, and this impact was additionally manifested by the severity of smoking and cumulative antiretroviral treatment (cumul. ART) (Table 5).

The TBW\% parameter had a significant impact on the occurrence of advanced lipodystrophy syndrome, and the severity of smoking and cumulative ARV treatment also had an impact (Table 6).

\section{Discussion}

An analysis of the body composition using electrical bioimpedance is considered a safe, non-invasive and reliable test method with high reproducibility. It is used in the care of patients with chronic diseases leading to disorders of body composition with accompanying metabolic abnormalities, as well as in healthy subjects [12]. In a multicentre study of the general population, aged 12-94 years, of both sexes and two races, Sun et al. evaluated the usefulness of se-
Table 6. Risk factors and percentage content of total body water (TBW\%) as variables in the study of individual impacts on the occurrence of advanced lipodystrophy. Logistic regression

\begin{tabular}{|c|c|c|c|}
\hline Variable & OR & $95 \% \mathrm{Cl}$ & $p$-value \\
\hline \multicolumn{4}{|c|}{ Unadjusted model } \\
\hline Age & 1.0522 & 0.9938 to 1.1139 & 0.0805 \\
\hline Sex-Male & 0.7801 & 0.2348 to 2.5914 & 0.6852 \\
\hline Pack-years & 1.0206 & 0.9878 to 1.0545 & 0.2206 \\
\hline Hypertension & 1.4581 & 0.4950 to 4.2954 & 0.4938 \\
\hline HOMA-IR* & 1.3892 & 0.8631 to 2.2361 & 0.1759 \\
\hline Cumul. ART & 1.0613 & 1.0153 to 1.1093 & 0.0084 \\
\hline TBW\% & 1.1104 & 1.0014 to 1.2313 & 0.0469 \\
\hline \multicolumn{4}{|l|}{ Stepwise } \\
\hline Pack-years & 1.0323 & 1.0019 to 1.0637 & 0.0371 \\
\hline Cumul. ART & 1.0685 & 1.0231 to 1.1159 & 0.0028 \\
\hline TBW\% & 1.0957 & 1.0087 to 1.1902 & 0.0304 \\
\hline
\end{tabular}

*HOMA-IR - homeostasis model assessment of insulin resistance

Pack-years - smoking cigarettes, ART - antiretroviral therapy, TBW\% - percentage content of total body water

lected parameters of FFM and TBW, and described them as useful for large epidemiological studies, taking into account specific equations appropriate for age, gender and race [13]. In HIV-infected patients, different disorders in body composition may be found, which are related to various factors such as side effects of antiretroviral drugs leading to lipodystrophy; HIV infection and the accompanying chronic inflammation, malnutrition and cachexia. In clinical practice, the changes described above are usually assessed based on a physical examination, which is not objective. Therefore, it would be valuable to introduce a non-invasive and inexpensive method to daily medical practice that could objectify the assessment of these changes.

In our observation, patients infected with HIV had significantly higher percentage values of FFM, TBW and MM than patients in the control group. The FM value, expressed both as an absolute value and as a percentage, was significantly lower in the study group. Moreover, a linear relationship of the values of FM, FFM\%, TBW\% and MM\% upon the severity of lipodystrophy syndrome in HIV-positive patients has been demonstrated. In the LS2 subgroup of patients, significantly higher values of FFM\%, TBW\% and MM\% and significantly lower values of FM (both absolute values and as a percentage) compared to the LS1 subgroup have been demonstrated. Fat tissue percentage content (expressed as the FM\% parameter and TBW\%) had a significant relationship with clinically advanced lipodystrophy syndrome. A relationship has been recorded between the value of BIA parameters and the type of dystrophic changes. The most significant changes were observed in patients with lipoatrophy who had the lowest absolute and percentage values of FM and the highest values of FFM\%, MM\% and TBW\%. 
There are not many studies evaluating usefulness of BIA in HIV-positive patients with LS. Our results are particularly consistent with results of other researchers who describe the use of various parameters of BIA, depending on the study design and selection of the study group [5, 14-16]. In a cross-sectional study of HIV-infected patients and healthy persons, Kotler et al. were seeking appropriate predictive equations for the BIA parameters that could be used to assess the nutritional status and hydration of infected patients. It was found that the BIA method reflects well the changes in body composition. With the help of BIA, it was possible to observe the changes in FFM and TBW by more than $5 \%$ in the vast majority of subjects. The study results confirm the usefulness of FFM, TBW and BCM parameters measured using BIA as accurate and precise in the evaluation and monitoring of nutritional status in HIV-infected patients [14]. In another study, Kotler et al. reported a significant reduction in BCM in patients with AIDS, as a parameter associated with malnutrition. The authors used the term "BCM depletion", which indicated terminal cachexia and was a bad prognostic factor despite periods of stabilisation observed in the clinical status of patients [15]. In the assessment of malnutrition in HIV-infected patients, Ott et al. assessed the following parameters: TBW, BCM, ECM and the ECM/BCM index, and compared them with a control group of healthy individuals. They found a significant reduction in $\mathrm{BCM}$, an increase in $\mathrm{ECM}$, and higher values of the ECM/BCM index in the studied group of patients. These parameters were characterised by a significantly higher sensitivity than the assessment of body mass and BMI. In addition, a relationship was observed between a decline in the value of BCM and advanced malnutrition in HIV-infected patients [16]. Freitas et al. compared body composition in HIV-infected patients on cART according to 4 distinct phenotypes of body fat distribution. They found that BIA was an easy and useful tool to evaluate fat mass and FFM [17]. Pietiläinen et al. showed that BIA is a good method in assessing the amount or changes in fat mass, but of limited significance in measuring visceral fat [8].

In the literature, there are few publications available on the use of electrical bioimpedance in the diagnosis of lipodystrophy syndrome. In some publications, the authors make a comparison of the various diagnostic methods evaluating dystrophic changes. Batterham et al. compared BIA and DXA in a population of Australian patients infected with HIV. They showed a significant correlation between BIA and DXA for the values of FFM and FM, and concluded that the BIA method was appropriate for studying patients with HIV/AIDS [5]. Vassimon et al. showed that FM and FFM parameters were accurate in assessing LS in HIV-infected patients and as having a high degree of correlation with DXA test results [18]. Forrester et al. compared the values of FM and FFM assessed using BIA and DXA methods in groups of HIV-infected and uninfected men and women, and showed agreement with DXA and FFM test results in men, whereas in the subgroups of women, overstated values of FFM were recorded in the BIA study in relation to DXA.
Based on this study, it was concluded that BIA, as a method of greater availability and lower cost compared to DXA, can be used to analyse body composition with an acceptable level of error in the studied population [6]. The same results were obtained by Pérez-Matute et al. [19].

On the other hand, other researchers found that BIA, as a method for diagnostics of LS in HIV-infected patients, requires establishment of cut-off points and more definitions. Schwenk et al. compared the whole body and segmental BIA tests with anthropometric measurements and linear clinical scale used for assessing the severity of LS. In patients with dystrophic changes, higher extracellular and intracellular water values were recorded, which were correlated with $\mathrm{BMI}$ and the sex of patients. Longitudinal comparison of the BIA parameters showed a significant increase in these parameters for both groups of patients - with LS and those without dystrophy. In addition, the BIA test results did not differ significantly between the subgroups of patients with different types of dystrophic changes. The researchers found that the BIA methods, anthropometric measurements and the clinical linear scale need re-calibration in diagnosis of LS in HIV-infected patients [10].

Anthropometric parameters, such as BMI and hip circumference, were highly positively correlated with the FM\% and negatively with FFM\% and TBW\%. In addition, BMI and hip circumference weakly negatively correlated with MM\%. Waist circumference had a weaker correlation with FM\%, FFM\% and TBW\% than hip circumference. Patients infected with HIV and with metabolic syndrome had a higher value of FM\%, and lower TBW\% compared with infected patients without metabolic syndrome. Our results are in accordance with those obtained by other authors $[6,18,19]$. They indicate that BMI, hip and to a lesser extent waist circumference can be used in the assessment of LS.

In the present study, the values of BIA parameters were referenced to the method used in clinical trials, i.e. a subjective, medical assessment of the severity of lipodystrophy syndrome. Significant results of single and multivariate analyses indicate a relationship between specified BIA parameters and the severity of symptoms of LS.

\section{Limitations of the study}

The greater number of HIV-positive patients and more than one BIA examination could allow to conclude more precisely about the usefulness of BIA in the diagnostics of lipodystrophy syndrome. A low number of women hindered analysis of the influence of the gender on the results of BIA.

\section{Conclusions}

The results of this study support the potential usefulness of BIA as a non-invasive and low-cost diagnostic method for lipodystrophy syndrome. Parameters such as FM\%, FFM\%, TBW\% and, to a lesser extent, MM\% can be used to assess the severity of lipodystrophy. However, there are still no clearly defined criteria or definitions to diagnose LS 
in HIV-infected population. The parameters available in the BIA test require a larger study population and continuation of research in order to develop LS diagnostic criteria and to establish its usefulness in the diagnostics of dystrophy changes in HIV-positive patients receiving cART.

\section{Acknowledgment}

This publication is a part of the "WroVasc - Integrated Cardiovascular Centre" Project co-financed by the European Regional Development Fund, within the Innovative Economy Operational Program, 2007-2013, at the Regional Specialist Hospital, Research and Development Centre in Wroclaw.

\section{Conflict of interest}

The author's declared no potential conflicts of interest with respect to the research, authorship, and/or publication of this article.

\section{References}

1. Nguyen A, Calmy A, Schiffer V, et al. Lipodystrophy and weight changes: data from the Swiss HIV Cohort Study 2000-2006. HIV Med 2008; 9: 142-150.

2. Finkelstein JL, Gala P, Rochford R, et al. HIV/AIDS and lipodystrophy: implications for clinical management in resource-limited settings. J Int AIDS Soc 2015; 18: 19033.

3. Peterson S, Martins CR, Cofrancesco J Jr. Lipodystrophy in the patient with HIV: social, psychological, and treatment considerations. Aesthet Surg J 2008; 28: 443-451.

4. Omolayo O, Sealy PL. HIV lipodystrophy syndrome. Hosp Physician 2008; 44: 7-14.

5. Batterham MJ, Garsia R, Greenop P. Measurement of body composition in people with HIV/AIDS: a comparison of bioelectrical impedance and skinfold anthropometry with dual-energy $\mathrm{x}$-ray absorptiometry. J Am Diet Assoc 1999; 99: 1109-1111.

6. Forrester JE, Sheehan HM, Joffe TH. A validation study of body composition by bioelectrical impedance analysis in human immunodeficiency virus (HIV)-positive and HIV-negative Hispanic men and woman. J Am Diet Assoc 2008; 108: 534-538.

7. Degris E, Delpierre C, Sommet A, et al. Longitudinal study of body composition of $101 \mathrm{HIV}$ men with lipodystrophy: dual-energy $\mathrm{X}$-ray criteria for lipodystrophy evolution. J Clin Densitom 2010; 13: 237-244.

8. Pietiläinen KH, Kaye S, Karmi A, et al. Agreement of bioelectrical impedance with dual-energy X-ray absorptiometry and MRI to estimate changes in body fat, skeletal muscle and visceral fat during a 12-month weight loss intervention. Br J Nutr 2013; 109: 1910-1916.

9. Freitas P, Santos AC, Carvalho D, et al. Fat mass ratio: an objective tool to define lipodystrophy in HIV-infected patients under antiretroviral therapy. J Clin Densitom 2010; 13: 197-203.

10. Schwenk A, Breuer P, Kremer G, et al. Clinical assessment of HIVassociated lipodystrophy syndrome: bioelectrical impedance analysis, anthropometry and clinical scores. Clin Nutr 2001; 20: 243-249.

11. Kwiatkowska W, Knysz B, Arczyńska K, et al. Peripheral arterial disease and ankle-brachial index abnormalites in young and middle-aged HIV-positive patients in Lower Silesia, Poland. PLoS One 2014; 9: e113857.

12. Lewitt A, Mądro E, Krupienicz A. Podstawy teoretyczne i zastosowania analizy impedancji bioelektrycznej (BIA). Endokrynol Otyłość 2007; 3: 79-84.
13. Sun SS, Chumlea WC, Heymsfield SB, et al. Development of bioelectrical impedance analysis prediction equations for body composition with the use of a multicomponent model for use in epidemiologic surveys. Am J Clin Nutr 2003; 77: 331-340.

14. Kotler DP, Burastero S, Wang J, et al. Prediction of body cell mass, fat-free mass, and total body water with bioelectrical impedance analysis: effects of race, sex, and disease. Am J Clin Nutr 1996; 64 (3 Suppl): 489S-497S.

15. Kotler DP, Wang J, Pierson RN. Body composition studies in patients with the acquired immunodeficiency syndrome. Am J Clin Nutr 1985; 42: 1255-1265.

16. Ott M, Lembcke B, Fischer H, et al. Early changes of body composition in human immunodeficiency virus-infected patients: tetrapolar body impedance analysis indicates significant malnutrition. Am J Clin Nutr 1993; 57: 15-19.

17. Freitas P, Carvalho D, Santos AC, et al. Assessment of body fat composition disturbances by bioimpedance analysis in HIV-infected adults. J Endocrinol Invest 2011; 34: 321-329.

18. Vassimon H, Machado AA, Paula FJ, et al. Bioelectrical impedance as a tool to assess body composition in HIV infected men with lipodystrophy syndrome. Poster presentation PP351, S: 160.

19. Pérez-Matute P, Pérez-Martínez L, Blanco JR, et al. Multiple frequency bioimpedance is an adequate tool to assess total and regional fat mass in HIV-positive patients but not to diagnose HIV-associated lipoatrophy: a pilot study. J Int AIDS Soc 2013; 16: 18609. 